Lothar Karl und Thomas Pieruschka

\title{
Application Service Providing (ASP) \\ für SISIS-Sunrise-Lokalsysteme im \\ Bibliotheksverbund Bayern: ein Anwenderbericht
}

Zusammenfassung: Die Verbundzentrale des Bibliotheksverbundes Bayern bietet Bibliotheken mit SISIS-Sunrise-Lokalsystem ein Application Service Providing an. Dargestellt werden wesentliche Aspekte dieses Services aus Sicht und Erfahrung einer Anwenderbibliothek, der Universitätsbibliothek der Universität der Bundeswehr München.

Schlüsselwörter: Application Service Providing, SISIS-Sunrise, Bibliotheksverbund Bayern

Application Service Providing (ASP) for SISIS-Sunrise library management systems by the Bavarian Library Network: a user report

Abstract: The Network's IT-coordination centre offers Application Service Providing to libraries using SISIS-Sunrise library management systems. This report outlines the main features of this service as experienced by a member library, the library of the Universität der Bundeswehr München.

Keywords: Application Service Providing, SISIS-Sunrise, Bavarian Library Network

\section{Application Service Providing}

Beim „Application Service Providing“ (ASP) stellt ein Anbieter dem Kunden eine Dienstleistung über ein Datennetz zur Verfügung. Dabei liegt die Software auf einem Server des Anbieters, der Kunde greift über das Internet auf die Programme

Lothar Karl: lothar.karl@unibw.de Thomas Pieruschka: thomas.pieruschka@unibw.de 
zu und bezahlt dafür ein Entgelt. Inbetriebnahme und laufender Betrieb des Servers, Installation neuer Software-Versionen und -patches, Datenbank-Administration, Datensicherheit und Datensicherung liegen allein in der Verantwortung des Anbieters. In der Regel umfasst ASP auch zusätzliche Dienstleistungen im Bereich der angebotenen Anwendung(en), wie Nutzerberatung und Hotline.

Abgesehen von der Beschaffung notwendiger Datenend- und PeripherieGeräte entfallen für den Kunden Anschaffungs- und Betriebskosten für die Server-Hardware. Er ist von der Systemverantwortung befreit und reduziert die dafür notwendigen Sach- und Personalmittel.

\section{Ausgangslage im Bibliotheksverbund Bayern}

Im Bibliotheksverbund Bayern (BVB) wird der Katalog der bibliografischen Daten aller BVB-Bibliotheken zentral durch die Verbundzentrale administriert, die dafür notwendige Hard- und Software ist im Leibniz-Rechenzentrum (LRZ) der Bayerischen Akademie der Wissenschaften installiert und steht den BVB-Bibliotheken kostenfrei zur Verfügung. Ein Endnutzerinterface (OPAC) für das Bibliotheksklientel sowie Ausleihe und Erwerbung finden in den Lokalsystemen der einzelnen Bibliotheken statt, die von den Bibliotheken vom Anbieter lizenziert werden. Verbund- und Lokalsystem sind durch eine Schnittstelle, die SIKOM, verbunden, die die im Verbundkatalog vorgenommenen Änderungen automatisch ins jeweilige Lokalsystem überträgt.

Vor 2001 liefen die Lokalsysteme jeweils auf eigenen Rechnern der Hochschulen, meist der Bibliotheken selbst oder in den Rechenzentren. So hatte sich eine sehr heterogene Lokalsystem-Landschaft entwickelt: Zwar wurde im BVB Anfang der 1990er Jahre im Rahmen des Projekts SOKRATES ein einheitliches Lokalsystem (SISIS-Sunrise von OCLC, früher SISIS) in den Hochschulen eingeführt, jedoch mit erheblichen Unterschieden sowohl bei der Leistungsfähigkeit der Hardware als auch bei den Versionsständen der Programme. In den Bibliotheken wurden Personalressourcen aufgrund der verantwortungsvollen und arbeitsintensiven Administration des eigenen Lokalsystems (Beschaffung neuer Hardware, Betreuung des Betriebssystems, der Datenbank und des Bibliothekssystems des lokalen Servers der PC der Mitarbeitenden) gebunden, eventuell unterstützt durch das jeweilige Rechenzentrum. Die immer schnelleren Produktzyklen und die steigende Komplexität im Umfeld des IT-Betriebes stellten immer höhere Anforderungen an die Verantwortlichen.

Diese Ausgangssituation, verbunden mit der Auslegung der SISIS-Lokalsysteme gemäß dem Server-Client-Modell und der bereits zentralen Erschließung in 
der Verbundkatalogdatenbank, führte zur Etablierung des ASP für SISIS-Bibliotheken durch die BVB-Verbundzentrale (BVB-VZ).

\section{Das Application Service Providing des Bibliotheksverbundes Bayern}

Erster Nutzer dieser Dienstleistung war im Jahr 2001 die Fachhochschulbibliothek (FHB) Ansbach. Im Anschluss daran wurden die SISIS-Systeme aller weiteren bayerischen Fachhochschulbibliotheken übernommen. Es folgten verschiedene Spezialbibliotheken (u.a. 2005 die Bibliothek des Deutschen Museums in München) und die regionalen Staatlichen Bibliotheken.

Im Jahr 2006 nahmen die Universitätsbibliotheken Augsburg, München und Regensburg diesen Service der BVB-VZ zur Betreuung ihrer Lokalsysteme an. Seit 2007 stand die Nutzung des ASP auch außerbayerischen Bibliotheken zur Verfügung, 2009 machten die ersten Bibliotheken aus Nordrhein-Westfalen und dem Kooperativen Bibliotheksverbund Berlin-Brandenburg (KOBV) davon Gebrauch, Letztere im Rahmen der strategischen Allianz zwischen KOBV und BVB.

Aktuell laufen 55 SISIS-Systeme im ASP-Dienst der BVB-VZ (12 Universitätsbibliotheken, über 20 Bibliotheken der Hochschulen für Angewandte Wissenschaften, vier Staatliche Bibliotheken, ein gemeinsames System der BehördenBibliotheken, über zehn Spezial- und sonstige Hochschulbibliotheken sowie zwei Öffentliche Bibliotheken). Durch die gemeinsame Nutzung von Lokalsystemen werden damit über 100 Bibliotheken mit Lokalsystemfunktionen versorgt.

Die gesamte Server-Hardware ist im LRZ in Garching bei München aufgestellt. Die Betriebssysteme sowie die Basis-Software werden durch das LRZ installiert und betreut, ebenso die Datensicherung. Die Datenbanksysteme und die SISIS-Lokalsysteme hingegen werden von Mitarbeitern und Mitarbeiterinnen der BVB-VZ betreut. Die Datenverbindung erfolgt über das Wissenschaftsnetz, für einen möglichen Ausfall steht eine zusätzliche Netzanbindung bereit.

Die Systeme laufen auf insgesamt 21 virtuellen Rechnern, wobei mehrere kleinere Lokalsysteme sich einen Rechner teilen. Dazu kommen 55 virtuelle Rechner als Frontend für die Webkataloge (OPAC) sowie einzelne Testsysteme je nach Bedarf. Die Systeme und Datenbanken der einzelnen Bibliotheken sind dabei völlig getrennt und voneinander unabhängig. Betriebssystem und SISISLokalsysteme befinden sich auf einem möglichst einheitlichen aktuellen Versionsstand.

Folgende Aufgaben haben die Bibliotheken im Rahmen des ASP an die BVB-VZ abgegeben bzw. werden von dieser übernommen: 
- Neuinstallationen und Inbetriebnahme von Lokalsystemen,

- Durchführung der Versionsupdates (nach Test in BVB-VZ),

- Datenbanksicherungen,

- Einrichtung der Server für Ausleihe/Katalogisierung/Erwerbung, OPAC (avserver, catserver, opserver),

- Zentrale Grundkonfiguration der Systeme,

- Konfiguration der SISIS-Sunrise-Administration (siadmin) mit Beratung bei Parameter- Einstellungen jeder einzelnen Bibliothek,

- Übernahme der Systemverwaltung,

- Feste Ansprechpartner für die Bibliotheken,

- Betreuung des laufenden Betriebes durch erfahrene Mitarbeitende der BVB-VZ in der First-Level-Hotline,

- Bereitstellung von Testsystemen,

- Fakultativ: Übernahme der Jahresarbeiten.

Die IT-Verantwortlichen der Bibliotheken bleiben Ansprechpartner der BVB-VZ und werden in der Administration ihrer Lokalsysteme nicht eingeschränkt. Sie erhalten eine bibliotheksspezifische Kennung für ihre Datenbank, allerdings keine root(super user)-Kennung für das Betriebssystem. Diese bleibt den Systembetreuern bei der BVB-VZ und im LRZ vorbehalten. Bei Bedarf werden der bibliotheksspezifischen Kennung jedoch für bestimmte Funktionen root-Rechte eingeräumt.

Die erste Voraussetzung für die Inanspruchnahme des ASP durch eine Bibliothek ist ein gültiger Software-Pflegevertrag für SISIS-Sunrise mit der Firma OCLC. Die zweite Voraussetzung ist die Unterzeichnung der Leistungsvereinbarung der BVB-VZ für den ASP-Dienst sowie die jährliche Entrichtung des Entgelts auf Basis des ASP-Kostenmodells im BVB. Diese ASP-Vereinbarung umfasst die genannten Services, nicht jedoch die Bereitstellung von Software-Lizenzen, Updates und Second-Level-Support durch OCLC.

Die Verantwortung für den Datenschutz verbleibt bei der jeweiligen Bibliothek. Hierfür sind gesonderte Regelungen zu treffen, z. B. ein mit dem LRZ abgestimmtes Sicherheitskonzept für den Betrieb der Anwendungen und Rechner.

Die ASP-Kosten werden nach der Anzahl der Titeldatensätze im lokalen Katalog und der Anzahl der eingesetzten SISIS-Sunrise-Komponenten (z. B. Ausleihe, Erwerbung) jeder nutzenden Bibliothek errechnet. Hinzu kommen weitere Kosten für die Nutzung der Hardware im LRZ. Der Gesamtbetrag wird auf der Basis der Bestandsgröße zu Jahresbeginn und zur Jahresmitte berechnet und jeweils für das folgende Halbjahr in Rechnung gestellt. 


\section{Umstellung des Lokalsystems der UB Uni Bw M auf ASP}

Die Universität der Bundeswehr München (UniBw M) ist eine von zwei Universitäten $^{1}$, die im Jahr 1973 vom Bundesministerium der Verteidigung eingerichtet wurden. Das Studium an einer dieser beiden Universitäten ist seitdem fester Bestandteil der Offiziersausbildung. Unterhaltsträger ist die Bundesrepublik Deutschland. Aufgrund der Kulturhoheit der Länder liegt die UniBw M hochschulrechtlich jedoch im Zuständigkeitsbereich des Freistaats Bayern. Das Studium und alle Abschlüsse sind mit denen der Landesuniversitäten absolut gleichwertig.

Dementsprechend war die Universitätsbibliothek der UniBw M (UB UniBw M) von Anfang an Teil der bayerischen Bibliothekslandschaft. Auch hinsichtlich der IT orientierte sich die UB UniBw M immer stark an den bayerischen Gegebenheiten: Im Jahr 1996 wurde die Bibliotheks-IT auf SISIS umgestellt, das StandardLokalsystem des BVB, bestehend aus den Komponenten Katalogisierung, Erwerbung, Ausleihe und OPAC. Allerdings wurde das System ohne Verbindung zur Verbunddatenbank betrieben. Die Titelaufnahmen wurden direkt mit der Katalog-Komponente von SISIS erstellt.

Im Jahr 2006 wurde die UB UniBw M als offizielles Mitglied in den Bibliotheksverbund Bayern aufgenommen. Voraussetzung für die Inanspruchnahme der Dienstleistungen des Verbundes war die Einspielung der bibliografischen Daten der UB in die Verbunddatenbank.

Im Folgejahr wurde von der UB UniBw M ein neuer Rechner für das Lokalsystem beschafft, im Rechenzentrum² der UniBw M (RZ UniBw M) aufgestellt, aber nach wie vor von dem IT-Referat der UB betrieben.

Dieser Rechner war im Jahr 2011 technisch veraltet und es war durch mehrere kleinere Hardware-Defekte bereits zu Systemausfällen gekommen. Als Ersatz wurden drei Möglichkeiten geprüft:

- Beschaffung eines neuen Rechners sowie des Betriebssystems, der Datenbank und der Anwendungsprogramme bei OCLC sowie Beauftragung von OCLC mit der vollständigen Neuinstallation des Systems; weitere Betreuung durch die UB UniBw M wie bisher.

1 Die zweite ist die Helmut-Schmidt-Universität (HSU) in Hamburg. Die UB HSU ist Mitglied im Gemeinsamen Bibliotheksverbund.

2 Unterbrechungsfreie Stromversorgung, entsprechende Klimatisierung und Brandschutz. Datensicherung durch den Backup-Service des RZ, ansonsten keine Systembetreuung durch das RZ-Personal. 
- Einrichtung eines virtuellen Servers im RZ UniBw M. Migration der Datenbank und Installation der Anwendungsprogramme durch die Firma OCLC. Serverbetrieb durch RZ UniBw M, Betreuung von SISIS-Sunrise durch die UB UniBw M.

- Nutzung der Dienstleistung ASP der BVB-VZ gegen Kostenübernahme.

Für eine Inanspruchnahme des ASP sprachen neben den bereits angeführten Gründen vor allem die positiven Erfahrungen anderer Bibliotheken. Auch die Kostenabschätzung sowie die Überprüfung der rechtlichen und technischen Voraussetzungen führten zur Entscheidung zugunsten des ASP-Dienstes. Das RZ UniBw M war frühzeitig in dieses Vorhaben eingebunden und gewährte der UB UniBw M die notwendige Unterstützung für die Netzanbindung zum LRZ. So wurde im Mai 2012 die Leistungsvereinbarung zwischen der UniBw M und der BVB-VZ mit Vertragsbeginn Juli 2012 geschlossen.

Bereits im Juli 2012 erstellte die BVB-VZ als Provider mit einer Kopie der Datenbank der UB UniBw M ein Testsystem und führte erste Prüfungen durch. Als Termin für den tatsächlichen Umstieg war unter Berücksichtigung der langfristigen Planung der BVB-VZ der November 2012 vorgesehen. Bis dahin sollten die notwendigen Vorbereitungen auf beiden Seiten abgeschlossen sein.

Die gesamte Planung wurde jedoch hinfällig, als am 26. Juli 2012 ein möglicherweise erfolgreicher Hackerangriff auf den Bibliotheksrechner erfolgte, dessen Folgen nicht einschätzbar waren. Nach den Sicherheitsrichtlinien des RZ UniBw M wurde dieser Rechner sofort vom Netz genommen. Ohne Lokalsystem stand der gesamte Bibliotheksbetrieb nahezu still. Eine unvermeidbare vollständige Neuinstallation von Betriebssystem, Datenbank und Anwenderprogrammen stand wenige Monate vor der Inanspruchnahme des ASP-Dienstes bevor. In jedem Fall musste eine schnelle Lösung gefunden werden.

Die BVB-VZ war grundsätzlich dazu bereit, den ohnehin geplanten Umstieg auf ASP vorzuziehen. Sie war jedoch durch ihre langfristige Zeitplanung gebunden, in der bereits Termine für Versionsumstiege o. ä. anderer Bibliotheken verbindlich vereinbart waren.

Unmittelbar stand das Update der Lokalsysteme der Regensburger Wissenschaftlichen Bibliotheken von der SISIS-SunRise Version 3.7 auf 4.1 bevor. Angesichts der Notlage der UB UniBw M verzichteten die Regensburger Bibliotheken freundlicherweise auf ihren vorgesehenen Termin. Diese Entscheidung ist umso höher zu bewerten, als es sich hier um ein aufwändiges Versions-Update handelte, für das bereits umfangreiche Vorarbeiten geleistet und ein längerer Zeitraum eingeplant worden waren.

Die Mitarbeitenden der BVB-VZ richteten nun mit Nachdruck ein funktionierendes System für die UB UniBw M ein. Zunächst wurde auf Basis des Testsystems 
ein Lokalsystem auf aktuellem Versionsstand eingerichtet, in das eine Kopie der Datenbank der UB UniBw M eingespielt werden konnte. Anschließend wurden die den Erfordernissen der UB UniBw M entsprechenden Bibliotheksparameter konfiguriert und die wesentlichen Anpassungen des InfoGuide-OPACs vorgenommen. Parallel dazu stellte das RZ UniBw M in direktem Kontakt zum LRZ die erforderliche VPN-Verbindung her.

Durch das große Engagement aller Beteiligten konnte am 8. August 2012, nach nur 13 Tagen Unterbrechung, der Bibliotheksbetrieb mit neuem Lokalsystem und ASP-Umstieg in vollem Umfang wieder aufgenommen werden. Der Betrieb des Lokalsystems im ASP lief von Anfang an störungsfrei.

Die UB UniBw M dankt an dieser Stelle allen für die uneingeschränkte Hilfe und Unterstützung!

\section{Betrieb und Erfahrungen}

Das System der UB UniBw M ist nach ASP-Standard auf zwei virtuelle Rechner verteilt: auf den Basisrechner mit Datenbank und Anwendungsservern und einen Rechner in der OPAC-Zone als Frontend für den InfoGuide als Endnutzerzugang.

Die BVB-VZ richtet sämtliche Anwendungsdrucker ein und betreut diese, ferner überwacht sie die SIKOM-Schnittstelle. Auch bei der Umsetzung von lokalen Besonderheiten wird die UB UniBw M nachhaltig von deren Mitarbeitenden unterstützt. Skripte, die einen Zugriff auf die Datenbank benötigen, z. B. für das Erzeugen der Benutzerausweise oder der Neuerwerbungslisten, wurden dadurch problemlos eingebunden. Auch die Anbindung von „Crystal Reports“ klappt fehlerfrei. Termine für systemrelevante Arbeiten werden schnell und formlos vereinbart, z. B. wurde seit August 2012 mehrfach ein Wörterbuchneuaufbau durchgeführt. Deutlich positiv für die UB UniBw M wirkt sich die Übernahme der Jahresarbeiten ,Ausleihe“ durch die BVB-VZ während der Schließungszeit der UB UniBw M zum Jahreswechsel aus: Damit steht am ersten Öffnungstag des neuen Jahres ein funktionierendes System bereit.

Nach wie vor bleiben jedoch, trotz dieser Unterstützung, die Mitglieder des IT-Referates die ersten bibliotheksinternen Ansprechpartner bei Störungen oder Fragen zum Bibliothekssystem. Diese können sich jedoch bei Bedarf immer an die Firstlevel-Hotline der BVB-VZ wenden, die schnell und zielführend Hilfe gewährt. Bei Fragen zu Software-Problemen übernimmt die BVB-VZ, sollte dies nötig sein, auch die Kommunikation und Abstimmung mit OCLC.

In der bisherigen ASP-Anwendungszeit wurde auch die Datensicherung auf die Probe gestellt: Im Sommer 2013 führte eine fehlerhafte, sehr umfangreiche 
Datenaufnahme von Studienanfängern und -anfängerinnen zu Inkonsistenzen in der Datenbank. Durch Einspielen einer Datenbanksicherung durch die BVB-VZ wurden diese jedoch problemlos bereinigt.

\section{Fazit}

Das „Application Service Providing“ hat unsere Erwartungen erfüllt. Die eingangs beschriebenen Vorteile dieses Dienstes haben sich in der Praxis bewährt. Die SISIS-Anwendungen laufen sehr zuverlässig und tragen so dazu bei, den Kundinnen und Kunden der UB UniBw M eine optimale Literaturversorgung zu gewährleisten.

Nähere Informationen zur Anwendungsbetreuung des BVB:

http://www.bib-bvb.de/web/sisis-lokalsysteme/fur-bibliotheken

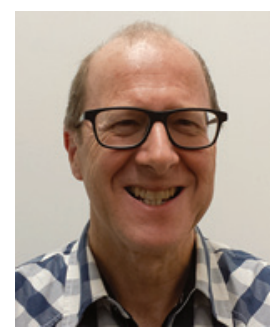

\section{Lothar Karl}

Universitätsbibliothek der Universität der Bundeswehr München Werner-Heisenberg-Weg 39

85577 Neubiberg

E-Mail: lothar.karl@unibw.de

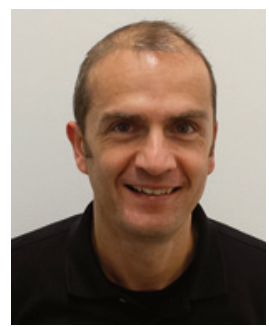

\section{Thomas Pieruschka}

Universitätsbibliothek der Universität der Bundeswehr München Werner-Heisenberg-Weg 39

85577 Neubiberg

E-Mail: thomas.pieruschka@unibw.de 